Spatial modulation of above-the-gap cathodoluminescence in InP nanowires

This content has been downloaded from IOPscience. Please scroll down to see the full text. 2013 J. Phys.: Condens. Matter 25505303

(http://iopscience.iop.org/0953-8984/25/50/505303)

View the table of contents for this issue, or go to the journal homepage for more

Download details:

IP Address: 93.180.53.211

This content was downloaded on 28/11/2013 at 06:56

Please note that terms and conditions apply. 


\title{
Spatial modulation of above-the-gap cathodoluminescence in InP nanowires
}

\author{
L H G Tizei ${ }^{1,2}$, L F Zagonel ${ }^{1}$, M Tencé ${ }^{2}$, O Stéphan ${ }^{2}$, M Kociak ${ }^{2}$, \\ T Chiaramonte ${ }^{3}$, D Ugarte ${ }^{1}$ and M A Cotta ${ }^{1}$ \\ ${ }^{1}$ Instituto de Física Gleb Wataghin, Universidade Estadual de Campinas-UNICAMP, \\ 13083-859 Campinas, SP, Brazil \\ ${ }^{2}$ Laboratoire de Physique des Solides, Université Paris-Sud, CNRS-UMR 8502, F-91405 Orsay, France \\ ${ }^{3}$ Universidade Federal de São João del Rei UFSJ, 36301-160 São João del Rei, MG, Brazil \\ E-mail: luiz.galvao-tizei@u-psud.fr
}

Received 26 June 2013, in final form 30 September 2013

Published 25 November 2013

Online at stacks.iop.org/JPhysCM/25/505303

\begin{abstract}
We report the observation of light emission on wurtzite InP nanowires excited by fast electrons. The experiments were performed in a scanning transmission electron microscope using an in-house-built cathodoluminescence detector. Besides the exciton emission, at $850 \mathrm{~nm}$, emission above the band gap from 400 to $800 \mathrm{~nm}$ was observed. In particular, this broad emission presented systematic periodic modulations indicating variations in the local excitation probability. The physical origin of the detected emission is not clear. Measurements of the spatial variation of the above-the-gap emission points to the formation of leaky cavity modes of a plasmonic nature along the nanowire length, indicating the wave nature of the excitation. We propose a phenomenological model, which fits closely the observed spatial variations.
\end{abstract}

(Some figures may appear in colour only in the online journal)

\section{Introduction}

The understanding of the optical properties of materials at the nanometer scale is of fundamental importance, given the large interest in nanometer-sized structures (such as quantum dots, quantum wells, nanowires, etc) due to the possibility of designing new devices or of observing new physical phenomena [1-7]. In particular, a wide range of applications of III-V semiconductors is being pursued in light emission and detection: GaN lasers [8], InP/InAsP light emitting diodes [9], GaAs single-photon sources [10] and photodetectors [11]. It is known that the properties of nanoscale materials can be drastically influenced by size and surface to volume ratio. For example, photoluminescence from $\mathrm{Si}$, an indirect band gap material, becomes effective at nanometric scale particles $[12,13]$. Moreover, new absorption modes are possible in metallic nanoparticles due to the appearance of surface plasmons [14]. Hence, the measurement of spatially resolved optical properties of nanomaterials can reveal new, unexpected effects.
In this work, we report the spatially resolved measurement of cathodoluminescence from InP nanowires in a scanning transmission electron microscope (STEM). Our experimental apparatus is explained in section 2. In these experiments, we have observed two different emissions, the exciton at $1.46 \mathrm{eV}$ and a wide band between 1.55 and $3.1 \mathrm{eV}$, described in section 3. A similar cathodoluminescence signal has been observed by Yamamoto et al [15] but without spatial distribution information. We present the analysis of the spatial variation of this emission in section 4 . This analysis, through a phenomenological model, gives insight into the wave nature of the excitation from which it originates and the formation of electromagnetic modes in the nanowire. Above-the-gap emission is not usually expected and has not been extensively studied. This kind of luminescence can be related to the formation of electromagnetic surface excitations such as surface polaritons [14] or surface exciton polaritons (SEP) [16].

The characterization of electromagnetic surface modes with light sub-wavelength resolution is fundamental to the understanding of light emission in sub-wavelength objects, 
such as nanowires. These modes may drastically influence the emission probability of an emitter embedded in such nanowires. For this reason, knowledge of the modal spatial distribution and the emitter position is necessary. We believe our results give new insights into how to access the previous point.

\section{Experimental details}

We have measured the cathodoluminescence signal of InP nanowires using an in-house-built light collection system [17] installed in a scanning transmission electron microscope (STEM). The collection system (nanoCL system) has a high efficiency, being capable of detecting single-photon emitters $[18,19]$. The microscope used was a VG HB 501 operated at $60 \mathrm{kV}$ and at $100 \mathrm{kV}$, which is equipped with a field emission electron gun and a cold sample stage (150 K). Typically, an approximately $1 \mathrm{~nm}$ wide focused electron beam is used to excite the material. The light output from the CL system was coupled to a light spectrometer, which is equipped with a Peltier cooled charge coupled device camera. Light was coupled to the spectrometer using a bundle of optical fibers with $200 \mu \mathrm{m}$ core radius. The experiments performed do not allow the determination of the position and direction of the emitted light. Only the excitation position can be controlled by scanning the electron beam. In this sense, the spatial resolution is achieved by the selective excitation of different regions of the sample, which can be as small as $5 \mathrm{~nm}$ [20] and is typically below $100 \mathrm{~nm}$ [18].

InP nanowires have been grown by chemical beam epitaxy on GaAs [001] substrates using gold nanoparticles as catalysts by the vapor-liquid-solid method [21,22]. Growth precursors were trimethyl indium (TMI) with hydrogen $\left(\mathrm{H}_{2}\right)$ as carrier gas and thermally decomposed phosphine $\left(\mathrm{PH}_{3}\right)$. Electron diffraction measurements on a transmission electron microscope show that the nanowires are grown in the wurtzite structure in the [0001] direction. This structure is typically observed in InP nanowires (figure 1(a)) even though it is not the lowest energy structure for bulk InP (for which is the zinc blende). The detailed origin for this structural change is still debated. But the wurtzite phase is usually observed in InP nanowire growth. However, it is known that many growth parameters, such as temperature, substrate quality, precursor pressures, catalyst metal and size, nanowire diameter and doping, can play a role in determining the dominant crystalline phase in these nanowire [23, 24].

\section{Experimental results and discussion}

\subsection{General cathodoluminescence characteristics}

A general analysis of the CL emission from InP NWs shows two kinds of emission (figure 1(b)): a sharp and intense emission at around $850 \mathrm{~nm}(1.46 \mathrm{eV})$ and a broad luminescence band above the gap between 1.55 and $3.1 \mathrm{eV}$.

The first emission is the one expected for the exciton from InP with the wurtzite structure [25-27]. The same excitonic emission has been observed (at a higher energy, $1.488 \mathrm{eV}$ )
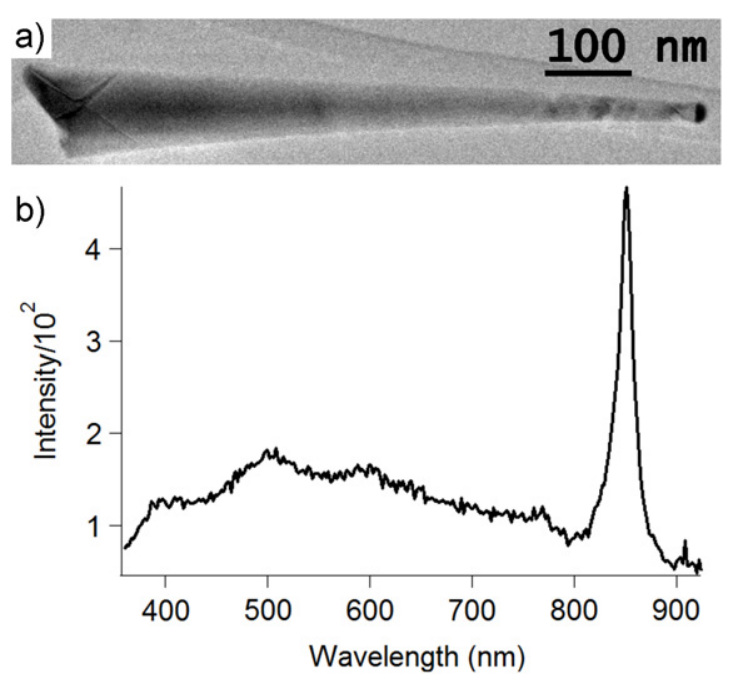

Figure 1. (a) Typical transmission electron microscopy image of a nanowire, showing a truncated cone geometry. (b) General cathodoluminescence spectrum of a typical InP nanowire with the wurtzite structure. Two emissions can be seen: (1) the excitonic emission at $850 \mathrm{~nm}, 1.46 \mathrm{eV}$, and (2) a wide band between 400 and $800 \mathrm{~nm}$.

by micro-photoluminescence performed in wurtzite InP nanowires grown in similar conditions [27]. This difference in energy is explained by the higher temperature used in the CL experiment $(150 \mathrm{~K})$ with respect to the PL one $(6 \mathrm{~K})$. In any case, the observed value is larger than that expected for zinc-blende InP $(1.4 \mathrm{eV})$. The exciton emission has not been observed in all NWs. Of those that presented it, some went dark after a few seconds or minutes of electron irradiation, while others continued to emit even after $30 \mathrm{~min}$ of exposure. These observations point out that the exciton emission may be quenched by the creation of defects, due to direct electron beam damage or to heating, possibly at the surface. One possibility is phosphorus desorption by knock-on damage. The different time scales may be linked to different heating extraction rates due to better contact to the support film (such behavior is known to occur in PL experiments in similar NWs) or to different surface quality of the NWs (thickness of the amorphous oxide covering the NWs, for example). Moreover, as will be shown later on (section 3.2), the maximum of the exciton emission occurs close to the thickest tip (arrow on figure 2), but away from the surface, indicating that the surface may play a role in quenching light emission.

The second emission, a large band above the InP band gap (from $400 \mathrm{~nm}, 3.1 \mathrm{eV}$, to $800 \mathrm{~nm}, 1.55 \mathrm{eV}$ ) is not expected for bulk InP (zinc blende or wurtzite). A similar effect has been previously observed by Yamamoto et al [15] for InP NWs with the zinc-blende structure in CL experiments in a TEM. In this report, the radial quantum confinement of excitons was considered as a possible explanation for the above-the-gap luminescence. However, as the authors point out, this effect cannot play a role in the nanowire presented here, because their diameters are large $(100-300 \mathrm{~nm})$ in comparison to the exciton Bohr radius in $\mathrm{InP}(9.3 \mathrm{~nm}$ for zinc-blende InP [15]). As we will explain later (section 4), the luminescence observed is probably linked to lossy surface 


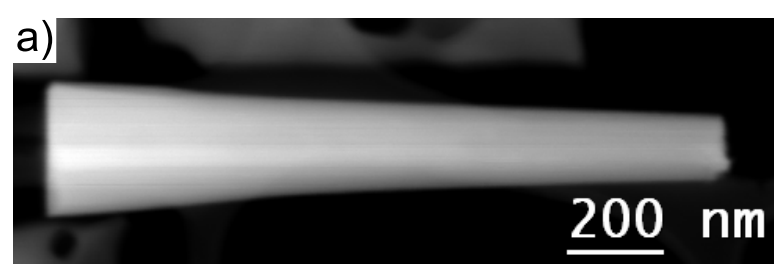

b)

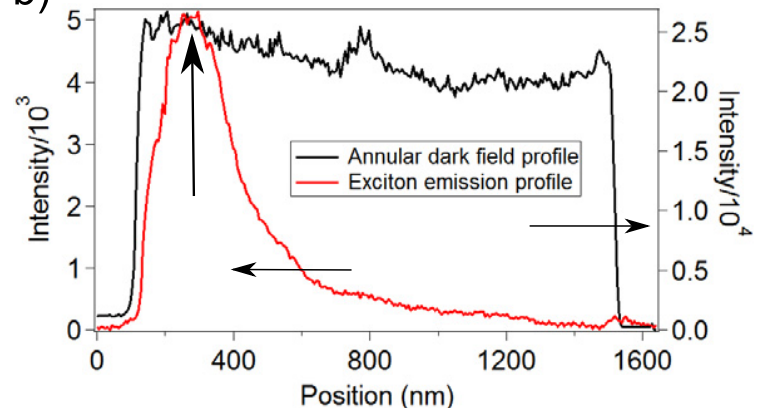

Figure 2. (a) Annular dark field image (ADF) of a nanowire showing the excitonic emission. (b) Line profiles along the nanowire growth direction of the integrated excitonic emission at $850 \mathrm{~nm}$ (red) and the ADF signal (black). The emission shows maxima close to the thickest tip of the nanowire, although away from the surfaces (the signal decreases close to the tip). The base of the nanowire (thickest region) is to the left. No modulation as a function of position was observed for this emission.

electromagnetic excitations. Detailed theoretical work is, however, required to definitely prove this point.

\subsection{Spatial modulation of cathodoluminescence}

To further analyze the physical origin of the observed above-the-gap luminescence, we have measured the spectral variation of the emission as a function of the position of the electron probe. We have been able to detect spatial variations of the emitted light, with a resolution limited by the material and excitation properties. In fact CL experiments are greatly adapted to the study of local variations in the optical properties at the nanometer scale of semiconductors and metals [17, 18, 28-30]. By scanning the electron probe we have acquired spectral images $[17,31]$, that is, images in which each pixel stores a full spectrum of the CL emission.

First of all, we show in figure 2 (see the caption) the spatial behavior of the exciton emission of a NW. In this figure, the abscissa represents the spatial direction along the $\mathrm{NW}$ axis. The exciton luminescence (at $850 \mathrm{~nm}$ ) follows the thickness variation of the NW to some extent. Moreover, surfaces seem to quench the luminescence (probably a carrier trap effect [32]), as the luminescence decreases before the thicker end of the NW.

In contrast to the exciton emission, we observe that the band above the gap changes as a function of position. In particular, we observe that at each position along a NW the spectrum contains one or more intensity maxima (as shown in figure 3). Interestingly, those maxima change in wavelength in a systematic fashion, which can be seen by the white (or dark) lines formed on the position versus wavelength $2 \mathrm{D}$ projection
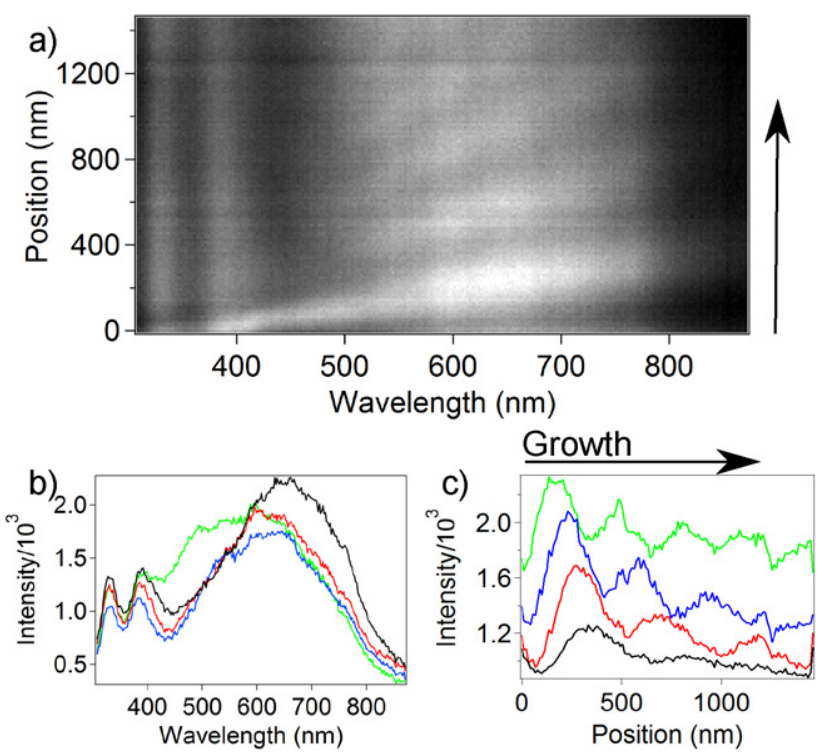

Figure 3. (a) Emission intensity as a function of position along the nanowire (vertical direction, the growth direction is shown by an arrow) and wavelength (horizontal direction). Oscillations of the emission intensity can be seen as a function of position, showing maxima at different wavelengths for each position along the NW. (b) General spectra at different positions along the nanowire showing the changes (the positions are $530 \mathrm{~nm}, 940 \mathrm{~nm}, 1180 \mathrm{~nm}$ and $1360 \mathrm{~nm}$ for the blue, red, black and green spectrum, respectively). (c) Intensity as a function of position along the NW for different wavelengths averaged in $11 \mathrm{~nm}$ wide windows: green $(560 \mathrm{~nm})$, blue $(650 \mathrm{~nm})$, red $(750 \mathrm{~nm})$ and black $(830 \mathrm{~nm})$.

and on the spectra shown in figures 3(a) and (b). In addition to that, the intensity at each wavelength oscillates along the NW, as depicted in figure 3(c), which shows the intensity integrated over three different energy windows along the NW (11 nm wide, 5 pixels, centered at $560 \mathrm{~nm}, 650 \mathrm{~nm}, 750 \mathrm{~nm}$ and $830 \mathrm{~nm}$ ). The NW whose luminescence is shown in figure 3 has a length of $2900 \mathrm{~nm}$. Only half of it is represented in the graphs shown.

The observed emission band has three peculiarities that deserve to be remarked:

- At each position of the electron beam a different spectrum is recorded.

- For each wavelength the spatial distribution of CL intensity oscillates with a period that is a monotonic function of the wavelength.

- The emission arises well above the gap.

The first two observations (i.e., the experimental observation of a dispersion relation) point to the confined wave-like character of the cause behind the observed second luminescence. These observations are evidence that the excitation probability changes as a function of position along the NW for each wavelength. In this sense, the presence of maxima is related either (1) to changes in the local radius of the NW or (2) to the absolute distance from the excitation position to the endpoints. This indicates the presence of confined modes, either longitudinal (cavity modes) or radial (whispering gallery modes (WGM) [28, 33]). In section 3.3 
we will discuss these two possibilities and demonstrate that the first case can explain the present results, while the second one cannot.

\subsection{Interpretation of the spatial modulation}

The observed dispersion makes it tempting to attribute it to the confinement of a wave. As we can approximate the NW shape as a truncated cone, two main sources of confinement might be invoked: either along the perimeter (WGM) or along the axis (cavity mode) of the NW.

The observation of WGM in $\mathrm{GaN}$ and $\mathrm{ZnO} \mathrm{NWs}$ has been reported before [28, 33]. The main idea is that modes containing an integer number of wavelengths are formed around the perimeter of the NW. In this way, for a given geometry, only some wavelengths will be permitted. Considering the typical tapered geometry of our NWs, we would expect that, as the radius of a NW varies, the permitted wavelength would change, giving rise to the observed smooth and continuous variation. However, the predicted behavior is not the correct one. That is, given a mode with a fixed number of nodes it is expected that, as the local mode size decreases (i.e., the perimeter which changes along the nanowire, figure 4(a)), the wavelength of this mode will decrease. In this way, for smaller radius we would expect a smaller wavelength for the maxima. What we observe in our experiments is the exact opposite: as we move along the growth direction of the NW (from bottom to top in figure 3) the radius decreases, but the emission maximum moves to longer wavelengths. A final point against this interpretation is that our NWs' radii range (even considering that the refractive index of zinc-blende $\mathrm{InP}$ is 3.5 at $1.5 \mathrm{eV}$ [34]) is below that which would allow a mode (we compared our nanowires to those of other reports [28, 33]). For this reason, no confinement along the perimeter is possible. This indicates that the modes we are observing are not related to WGM.

Let us thus consider the possibility of longitudinal cavity modes. In a perfect longitudinal cavity (i.e., a rectangular box), only a few modes would be expected, that is, those for which a half-integer number of wavelengths fit the cavity. These modes would have nodes distributed discretely along the cavity. Evidently, that is not what we observe in our results, as we have light excitation over the whole band along the whole NW. The element missing in this case is the lossy nature of the cavity, as will be described in what follows.

To explain our observations we have used a model of plane waves propagating along a cavity, following reference [10]. In a cavity, stationary modes are formed due to the constructive interference of propagating waves in different directions; that is, at a given position $x$ in a cavity of length $L$ (figure 4(a)) two counter-propagating waves (with amplitude $A^{+}$and $A^{-}$) of wavelength $\lambda$ have relative phases such that their amplitudes interfere constructively and destructively in certain positions, forming standing waves. These amplitudes (' $\mathrm{t}$ ' and ' $\mathrm{b}$ ' mean tip and base, representing the two endpoints of the NW) are

$$
\begin{gathered}
A^{+}=A_{\mathrm{s}}+r_{\mathrm{b}} A^{-}, \\
A^{-}=A_{\mathrm{s}}+r_{\mathrm{t}} A^{+}
\end{gathered}
$$
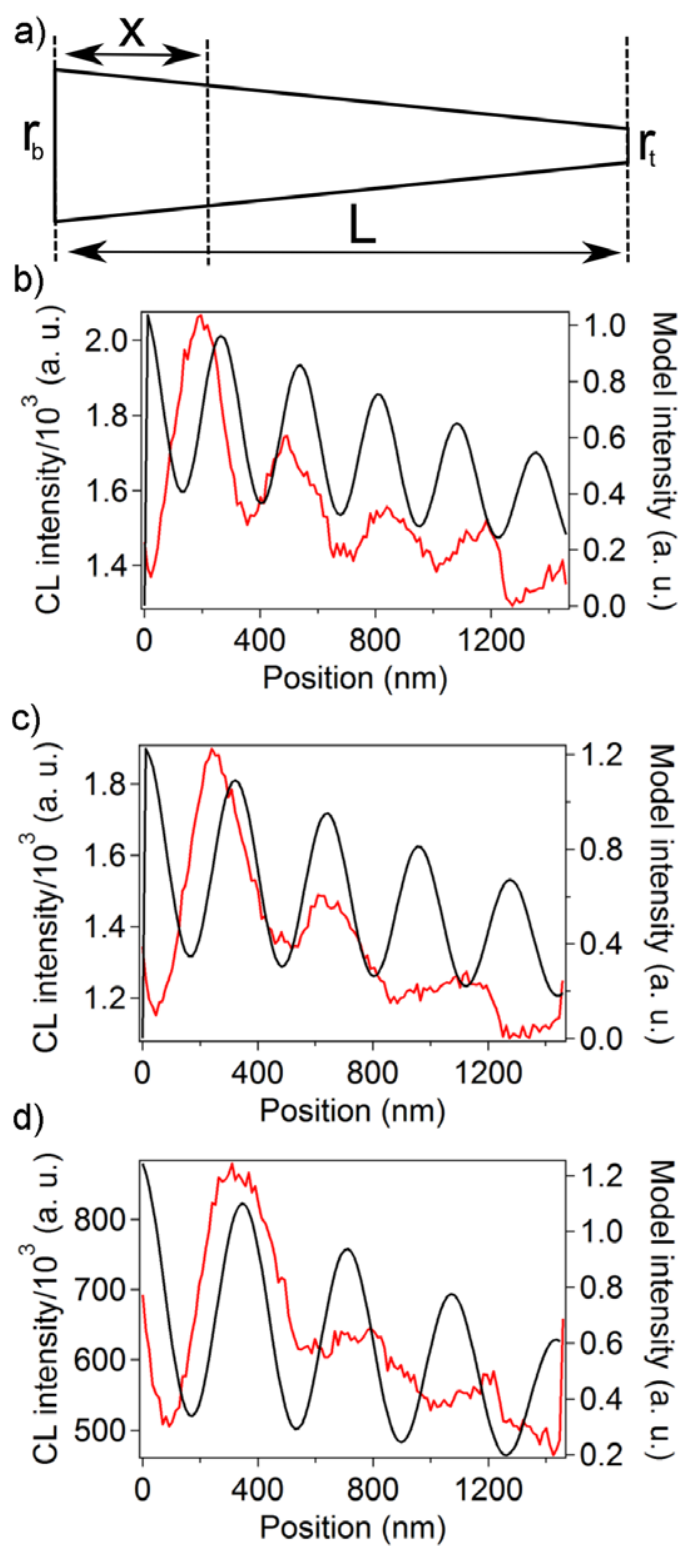

Figure 4. (a) Sketch of a NW describing the parameters use in the model. Note that the radius at each position along the nanowire varies. Measured intensity (red) versus intensity calculated from the model (black) for different wavelengths: (b) $600 \mathrm{~nm}$, (c) $700 \mathrm{~nm}$ and (d) $800 \mathrm{~nm}$.

where $r_{\mathrm{t}}=R_{\mathrm{t}}^{(1 / 2)} \mathrm{e}^{\left(\mathrm{i} \phi_{\mathrm{t}}\right)}$ and $r_{\mathrm{b}}=R_{\mathrm{b}}^{(1 / 2)} \mathrm{e}^{\left(\mathrm{i} \phi_{\mathrm{b}}\right)}$ are complex numbers which include the propagation phases $\phi_{\mathrm{t}}=2 \pi(L-$ $x) /\left(\lambda n_{\mathrm{eff}}\right)$ and $\phi_{\mathrm{b}}=2 \pi x /\left(\lambda n_{\mathrm{eff}}\right)$, where $x$ is the position along the nanowire and $n_{\text {eff }}$ is the wave propagation constant. $R_{\mathrm{t}}$ and $R_{\mathrm{b}}$ are the reflectivity of each end surface. The probability of exciting at a particular wavelength will depend on the square of the total amplitude, $A=A_{\mathrm{s}}+A_{\mathrm{t}} . L, \lambda$ and $n_{\mathrm{eff}}$ were chosen in the model to fit the observed oscillations, as discussed in what follows.

As expected, for a given $L$ (with $R_{\mathrm{t}}=R_{\mathrm{b}}=1$ ) only some $\lambda$ are allowed, giving rise to localized maxima in the intensity of the total electric field. This occurs because of the boundary conditions. However, if somehow these boundary conditions are modified, the behavior will be completely different. This 
can be achieved by changing the reflectivity of one or both end facets of the cavity. Reflectivity smaller than 1 is expected as some light will escape from the material; moreover, the ends of the NW have different diameters (one end is usually in the above $100 \mathrm{~nm}$ range while the other is usually below this) justifying the use of different reflectivities for each endpoint. Our hypothesis is that the emitted intensity is proportional to $|A|^{2}$, as expected for the electromagnetic local density of states [35]. The final total intensity in the model is normalized by the thickness of the nanowire at each position.

In figures 4(b)-(d) we show the comparison between the detected intensities and the model integrated in three wavelength ranges $(11 \mathrm{~nm}$ (5 pixels) windows centered at $600,700$ and $800 \mathrm{~nm})$. The parameters for the model in this calculation were $L=2900 \mathrm{~nm}, R_{\mathrm{b}}=0.9, R_{\mathrm{t}}=0.1$ and $n_{\text {eff }}=2.2 . L$ was measured from the experimental images. The shape of the curve does not depend much on $R_{\mathrm{b}}$ and $R_{\mathrm{t}}$. The values were taken to demonstrate the validity of the model. The last constant $n_{\mathrm{eff}}$, which defines the propagation phase, was chosen to approach the calculated oscillations as best as possible to the observed oscillations. This number is smaller than the refractive index of InP, which is 3.5 at $1.5 \mathrm{eV}$. A better agreement can be achieved by assuming that $n_{\text {eff }}$ changes as a function of $\lambda$. But as the nature of the excitation is not clear, it was uncertain which function should be used. For this reason we used a constant $n_{\text {eff }}$.

The relatively good agreement between the measured and the calculated intensity oscillations show that the proposed model captures some aspects of the observed effect, demonstrating the wave nature of the excitation. A complete model would need to take into account the correct nature of the excitation created and, with that, the proper values for the propagation phase constant. In fact, this value may change as a function of the radius of the nanowire, in analogy to the changes of the refractive index in waveguides with sizes comparable to the wavelength of light. Finally, as these modes need to be leaky to explain our results, the probability to emit photons is high.

\subsection{Origin of luminescence: surface plasmons}

As it occurs far above the band gap, the luminescence observed cannot be due to the decay of excitons created in the material by the electron beam. For the same reason the observed modes cannot originate due to guided light, as they would be heavily absorbed. Furthermore, possible quantum confinement effects cannot explain the large energy shifts because the size of the observed nanowires is much larger than the exciton Bohr radius, leading to confinement energies far smaller than $1 \mathrm{eV}$. This agrees with the interpretation presented by Yamamoto et al [15].

One possible explanation for the observed cathodoluminescence is the decay of electromagnetic surface excitations, such as surface polaritons [14] or surface exciton polaritons (SEP) [16]. It is worth noting that surface polaritons naturally couple to light, once symmetry conditions are met [36, 37]. This is particularly true for particles wider than the wavelength of light, such as the nanowires discussed here.
Moreover, less absorption is expected (in comparison to bulk modes) for surface excitations. However, detailed theoretical work is required to definitely prove this point. A deeper understanding of the physical origin of this excitation could be achieved by absorption measurements (such as electron energy loss spectroscopy). However, energy resolution of the order of a few meV (which is possible in our CL setup) or a few tens of milli-electron volts at most (and with a nanometer spatial resolution) would be needed, which cannot be achieved with current technology for electron energy loss spectroscopy.

\section{Conclusions}

We have observed exciton and above-the-gap emission from wurtzite InP nanowires excited by fast electrons. This luminescence shows intensity modulations along the nanowire, consistent with the formation of leaky cavity modes, as demonstrated by our phenomenological model. As such modes should be observed in other types of semiconductor nanowires, they deserve a full theoretical investigation.

\section{Acknowledgments}

This work has received support from $\mathrm{CNPq}$, from 2007/58962-1, 2008/55023-7, 2011/05989-5, São Paulo Research Foundation (FAPESP), from the National Agency for Research under the program of future investment TEMPOS-CHROMATEM with the reference no ANR-10EQPX-50 and from the European Union Seventh Framework Programme (No FP7/2007-2013) under Grant agreement no n312483 (ESTEEM2).

\section{References}

[1] Lu W and Lieber C M 2006 J. Phys. D: Appl. Phys. 39 R387

[2] Law M, Goldberger J and Yang P 2004 Annu. Rev. Mater. Res. 3483

[3] Samuelson Let al 2004 Physica E 25313

[4] Agarwal R 2008 Small 41872

[5] Chen S, Shehata C, Fradin R, Lapierre C, Couteau C and Weihs G 2007 Nano Lett. 72584

[6] Krogstrup P, Yamasaki J, Sorensen C B, Johnson E, Wagner J B, Pennington R, Aagesen M, Tanaka N and Nygard J 2009 Nano Lett. 93689

[7] Holmes J D, Johnston K P, Doty R C and Korgel B A 2000 Science 2871471

[8] Johnson J C, Choi H-J, Knutsen K P, Schaller R D, Yang P and Saykally R J 2002 Nature Mater. 1106

[9] Minot E D, Kelkensberg F, van Kouwen M, Louwenhoven L P, Zwiller V, Borgström M T, Wunnicke O, Verheijen M A and Bakkers E P A M 2007 Nano Lett. 7367

[10] Friedler I, Sauvan C, Hugonin J P, Lalanne P, Claudon J and Gérard J M 2009 Opt. Express 172095

[11] Pettersson H, Trägardh J, Persson A I, Landin L, Hessman D and Samuelson L 2006 Nano Lett. 6229

[12] Canham L T 1990 Appl. Phys. Lett. 571046

[13] Wolkin M V, Jorne J, Fauchet P M, Allan G and Delerue C 1999 Phys. Rev. Lett. 82197 
[14] Egerton R F 1996 Electron Energy-Loss Spectroscopy in the Electron Microscope (New York: Plenum)

[15] Yamamoto N, Bhunia S and Watanabe Y 2006 Appl. Phys. Lett. 88153106

[16] Chu M-W, Chen C-H, García de Abajo F J, Deng J-P and Mou C-Y 2008 Phys. Rev. B 77245402

[17] Zagonel L F et al 2011 Nano Lett. 11568

[18] Tizei L H G and Kociak M 2012 Nanotechnology 23175702

[19] Tizei L H G and Kociak M 2013 Phys. Rev. Lett. 110153604

[20] Zagonel L F, Rigutti L, Tchernycheva M, Jacopin G, Songmuang R and Kociak M 2012 Nanotechnology 23455205

[21] Wagner R S and Ellis W C 1964 Appl. Phys. Lett. 489

[22] Tizei L H G, Chiaramonte T, Ugarte D and Cotta M A 2009 Nanotechnology 20275604

[23] Glas F, Harmand J-C and Patriarche G 2007 Phys. Rev. Lett. 99146101

[24] Oliveiras D S, Tizei L H G, Ugarte D and Cotta M A 2013 Nano Lett. 13

[25] Mattila M, Hakkarainen T, Mulot M and Lipsanen H 2006 Nanotechnology 171580
[26] Mishra A et al 2007 Appl. Phys. Lett. 91263104

[27] Gadret E G et al 2010 Phys. Rev. B 82125327

[28] Nobis T, Kaidashev E M, Rahm A, Lorenz M and Grundmann M 2004 Phys. Rev. Lett. 93103903

[29] Vesseur E J R, de Abajo F J G and Polman A 2009 Nano Lett. 93147

[30] Storm K, Halvardsson F, Heurlin M, Lindgren D, Gustafsson A, Wu P M, Monemar B and Samuelson L 2012 Nature Nanotechnol. 7718

[31] Jeanguillaume C and Colliex C 1986 Ultramicroscopy 28252

[32] Yu P Y and Cardona M 2005 Fundamentals of Semiconductors (New York: Springer)

[33] Long J P, Flynn R A, Vurgaftman I, Simpkins B S, Mäkinen A J, Mastro M A and Pehrsson P E 2010 Appl. Phys. Lett. 97103105

[34] Palik E D 1985 Handbook of Optical Constants of Solids (New York: Academic)

[35] García de Abajo F J and Kociak M 2008 Phys. Rev. Lett. 100106804

[36] García de Abajo F J 1999 Phys. Rev. B 593095

[37] García de Abajo F J 2010 Rev. Mod. Phys. 82209 